Georgia State University

ScholarWorks @ Georgia State University

7-1-2010

\title{
Identification of Voters with Interest Groups Improves the Electoral Chances of the Challenger
}

Vjollca Sadiraj

Georgia State University

Jan Tuinstra

Georgia State University

Frans van Winden

Georgia State University

Follow this and additional works at: https://scholarworks.gsu.edu/excen_workingpapers

\section{Recommended Citation}

Sadiraj, Vjollca; Tuinstra, Jan; and van Winden, Frans, "Identification of Voters with Interest Groups Improves the Electoral Chances of the Challenger" (2010). ExCEN Working Papers. 81.

https://scholarworks.gsu.edu/excen_workingpapers/81

This Article is brought to you for free and open access by the Experimental Economics Center at ScholarWorks @ Georgia State University. It has been accepted for inclusion in ExCEN Working Papers by an authorized administrator of ScholarWorks @ Georgia State University. For more information, please contact scholarworks@gsu.edu. 
Identification of Voters with Interest Groups Improves the Electoral Chances of the Challenger

\author{
Vjollca Sadiraj, Jan Tuinstra and Frans van Winden
}

Experimental Economics Center, Andrew Young School of Policy Studies, Georgia State University

July 2010

Forthcoming in Mathematical Social Sciences 


\title{
Identification of Voters with Interest Groups Improves the Electoral Chances of the Challenger
}

\author{
Vjollca Sadiraj*, Jan Tuinstra ${ }^{\dagger}$ and Frans van Winden ${ }^{\ddagger}$
}

July 2010

\begin{abstract}
This short paper investigates the consequences of voters identifying with special interest groups in a spatial model of electoral competition. We show that, by effectively coordinating voting behavior, identification with interest groups leads to an increase in the size of the winning set, that is, the set of policy platforms for the challenger that will defeat the incumbent. Consequently, our paper points at a novel process through which interest groups can enhance the electoral chances of a challenger.
\end{abstract}

Keywords: Spatial voting models, electoral competition, winning set, interest groups.

JEL classification code: D71; D72.

\section{Introduction}

Special interest groups play an important role in political decision making (Richardson, 1994, Potters and Sloof, 1996, Congleton, Hillman, and Konrad, 2008). Regarding electoral competition - the focus of this study - a substantial number of theoretical studies have appeared over the last two decades trying to explain the influence of interest groups through campaign contributions, strategic information transmission, and the like (for surveys, see Austen-Smith 1994, Grossman and Helpman, 2001, van Winden, 2003, Prat, 2006). Thus far, however, the impact of interest groups on the political preferences and voting behavior of voters through the sociopsychological mechanism of identification has been neglected. This is in line with Akerlof and Kranton's (2000) more general observation that identity is "a missing motivation in economists' current description of organizations" (op. cit., p.10). Their seminal

*Department of Economics, Andrew Young School of Policy Studies, Georgia State University, vsadiraj@gsu.edu.

${ }^{\dagger}$ Department of Quantitative Economics and CeNDEF, University of Amsterdam, Amsterdam, The Netherlands, J.Tuinstra@uva.nl.

$\ddagger$ Department of Economics and CREED, University of Amsterdam, Amsterdam, The Netherlands, F.A.A.M.vanWinden@uva.nl. 
analysis suggests that this neglect is an important shortcoming of the present state of the art. Identity refers to a person's social category and self-image (e.g. being 'leftist', 'rightist', 'green', or 'red') and it: "describes gains and losses in utility from behavior that conforms or departs from the norms for particular social categories in particular situations" (op. cit., p.12). Identification with an organization means that the goals of that organization become important to the self, inducing people to behave in concert with these goals, even if social sanctions on deviant behavior are absent. To be politically effective, an important goal of a special interest group involved in electoral competition is the coordination of the voting behavior of its supporters ${ }^{1}$ on the political issue that the group stands for. ${ }^{2}$ For voters concerned about existing policies, supporting an interest group on an issue that is of particular concern to them seems a natural and even 'rational' thing to do in an environment that is complicated and where a single vote has (generally) no clout. Since negative experiences appear to have a relatively stronger impact on behavior (see e.g. Baumeister et al. 2001, and Offerman, 2002) one may arguably expect that especially dissatisfied and frustrated voters will try to link up with others feeling similarly. Interest groups can offer such voters a focal mechanism to express their concerns in a cost-effective way, through the alignment of voting behavior. The literature on identity namely suggests that those identifying with an interest group will, to a certain extent, give priority to the issue promoted by this group, which may imply a deviation from self-interest due to conformity effects (Shayo, 2009). Consequently, the issue at stake may gain more political clout than predicted by existing models of electoral competition. Moreover, there is plenty of empirical evidence suggesting that group membership affects preferences. Experiments have shown that even minimal groups, defined by an arbitrary color label like yellow or blue and with random assignment of individuals, affect behavior (Brewer, 1979, Tajfel and Turner, 1986, Schram and Sonnemans, 1996). In fact, the psychological theory of cognitive dissonance predicts that already the decision to join a group will tend to make the issues represented by that group relatively more attractive (Festinger and Aronson, 1968). Akerlof and Kranton (2000) have shown for various types of economic interactions that taking into account the phenomenon of identification - that is, incorporating identity into the utility function substantively changes the conclusions of previous economic analyses. The aim of this paper is to theoretically investigate the consequences of identification in a political economic context, using a standard spatial model of electoral competition (see e.g. Enelow and Hinich, 1984).

More specifically, our research question concerns the differential impact of the presence of interest groups on electoral competition if voters can support and identify with an interest group that furthers an issue of importance to them. Making the usual assumption of (weighted) Euclidean distance preferences in the absence of interest groups, essentially, identification in this context implies that voters will attach

\footnotetext{
${ }^{1}$ Note that identification with an interest group need not necessarily imply (registered) membership of that group. People may be supportive of an interest group's objectives without becoming a formal member. In this paper we will not distinguish between these two forms.

${ }^{2}$ For an empirical illustration, see Chong (1991, p.236): "Black organizations and institutions helped to coordinate the preferences and actions of those who supported the civil rights movement."
} 
extra weight or priority to the issue represented by the interest group. Furthermore, it will be assumed that the support of an interest group (and, thus, identification) is positively related with the level of dissatisfaction with the incumbent's policy. This assumption is supported by the ample evidence on the importance of discontent and frustration for collective action (see e.g. Kernell, 1977, Lau, 1982, Chong, 1991, Romer, 1996, Javeline, 2003). Identification based coordination of voting behavior then produces our main result which relates to the winning set, i.e. the set of policy platforms with which a challenger can defeat a given incumbent's policy position. Assuming for tractability a two-dimensional issue space and a uniform distribution of the voters ideal points over this space, we are able to show that the introduction of interest groups in this environment typically increases the winning set. Our demonstration provides a theoretical foundation for a similar observation made by Sadiraj, Tuinstra and van Winden (2006) on the basis of simulations with a more complex computational model. This result is particularly important if one takes into account that in practice political parties do not have complete information about voting behavior, urging them to use instruments like polls for acquiring information (see Sadiraj, Tuinstra and van Winden, 2006). Our result then suggests that in the presence of interest groups, it will be easier for the challenger to find a position defeating the incumbent. Or, put differently, the challenger's probability of winning the election will be enhanced by interest groups. As a further consequence, election outcomes may become more volatile.

The organization of this paper is as follows. Section 2 presents the spatial competition model with interest groups and our main result. The proof of the main result is outlined in Section 3, whereas the formal proofs of the results used there are relegated to the Appendix. Section 4 concludes with a further discussion of relevance and implications.

\section{Spatial competition and interest groups}

Two political parties, an incumbent and a challenger, are assumed to compete for votes by selecting certain policy platforms. Platforms are represented as points in an issue space. We take this issue space to be continuous and equal to $\mathcal{X}=B(O, K) \subset$ $\mathbb{R}^{2}$, that is, political parties can choose platforms on two dimensions from an open ball with center at the origin $O=(0,0)$ and radius $K>0 .{ }^{3}$ There is a continuum of voters where each voter $j$ is characterized by an ideal point $x^{j} \in \mathcal{X}$ and an issue weight vector or profile $s^{j} \in \mathcal{S}^{2}$ where $\mathcal{S}=\left\{s^{1}, s^{2}, \ldots, s^{r}\right\}$, with $\underline{s}=s^{1}<s^{2}<\ldots<s^{r}=\bar{s}$. Let $^{4} \underline{s}>0$ and, without loss of generality, $\bar{s}=1$. Suppose voters' ideal points are uniformly distributed over $\mathcal{X}$ and voters' weights are independently and identically distributed (and also independently from voters' ideal points) according to some

\footnotetext{
${ }^{3}$ We will return to this assumption in Section 4.

${ }^{4}$ As will become clear when we introduce interest groups, voters with weight 0 on one of the issues do not change their voting behavior when these interest groups enter the scene. Taking $\underline{s}>0$ is therefore an innocuous assumption. Moreover, the alternative assumption that strengths are continuously distributed on $[\underline{s}, \bar{s}]$ would lead to the same results.
} 
distribution on $\mathcal{S}{ }^{5}$ A voter's utility with respect to a certain policy outcome $y \in \mathcal{X}$ is given by the negative of the (weighted) Euclidean distance between this policy outcome and the voter's ideal point. Formally, utility for voter $j$ of policy outcome $y$ is given by ${ }^{6}$

$$
u^{j}(y)=-\left\|x^{j}-y\right\|_{s^{j}}^{2},
$$

where $\left\|x^{j}-y\right\|_{s^{j}}^{2} \equiv s_{1}^{j}\left(x_{1}^{j}-y_{1}\right)^{2}+s_{2}^{j}\left(x_{2}^{j}-y_{2}\right)^{2}$.

Let $y$ be the given position of the incumbent. ${ }^{7}$ In the absence of interest groups voters will vote for that candidate whose position will give him or her the highest utility according to (1). In case of a tie the voter will randomize, with equal probability, between the candidates. Now define by $W(y) \subset \mathcal{X}$ the set of policy platforms $z$ that are expected to attract (strictly) more votes than the incumbent's platform $y$. Clearly, the challenger's objective is to select a policy platform from $W(y)$. Notice that by definition $y \notin W(y)$ since a voter is indifferent between a challenger and the incumbent with identical policy platforms. An equilibrium point of the electoral competition game is defined as a platform $y^{*}$ for which the winning set is empty, i.e. $W\left(y^{*}\right)=\emptyset$. The uniform distribution of voters' ideal points over the issue space $\mathcal{X}$ implies that in our framework such an equilibrium point will indeed exist (cf. Plott, 1967 ) and is in fact given by the origin (this statement will be made more precise in Proposition 2 in Section 3). ${ }^{8}$ By $W(y ; s)$ we will denote the set of policy platforms defeating the incumbent's platform $y$ in the special case where all voters have weight vector $s$.

The next step is to incorporate interest groups in the model. We model a special interest group as being interested in the policy outcome with respect to only one of the two issues. Moreover, for tractability, we are going to investigate the situation where for every position on an issue voters can join an interest group and where all dissatisfied voters join an interest group. ${ }^{9}$ Before we describe how voting behavior is coordinated by the interest groups let us first determine how individual voters decide which interest group to join. An individual voter with ideal position $x=\left(x_{1}, x_{2}\right)$ is a potential member of two interest groups, the interest group on the first issue located at position $x_{1}$ and the interest group on the second issue located at position $x_{2}$. Which interest group this voter joins depends upon the incumbent's position and the

\footnotetext{
${ }^{5}$ That is, for all $r, t \in \mathcal{S}$ and all $v, w \in \mathcal{X}$ we assume that $\operatorname{Pr}\{s=(r, t)\}=\operatorname{Pr}\left\{s_{1}=r\right\} \operatorname{Pr}\left\{s_{2}=t\right\}$ and $\operatorname{Pr}\{s=(r, t) \mid x=v\}=\operatorname{Pr}\{s=(r, t) \mid x=w\}$.

${ }^{6}$ Notice that, following Enelow and Hinich (1984), we assume that preferences are separable. Our formulation implies that the indifference curves are ellipses with horizontal and vertical axes.

${ }^{7} \mathrm{It}$ is realistic to assume that the challenger is more flexible in choosing a platform, since $(\mathrm{s})$ he cannot be held responsible for the existing policy (see e.g. Kramer, 1977, and Kollman, Miller, and Page, 1992). For tractability, we use an idealization here.

${ }^{8}$ Note however, that this is not necessarily the case for finite samples of voters (see Tovey, 2010, for a discussion of distributional analysis of spatial voting models).

${ }^{9}$ Thus, there are many interest groups and an interest group exists at every position of each issue. Every voter has the possibility to join one of two of those interest groups, namely the interest group that has the same position as him/her on the first issue, or that interest group that has the same position as him/her on the second issue. As our focus is on the consequences of identification we keep this part of the model as simple as possible. (Note that our assumption does not imply that there is a seperate interest group for each position in the issue space, which would obviously make the concept void.)
} 
weights the voter attaches to the two issues. We assume that voters are more inclined to support an interest group on a particular issue the more distant the incumbent's policy position is from their own position on that issue (see the Introduction). ${ }^{10}$ Of course, the weight a voter attaches to the issues also comes into play. Hence we assume that, given the incumbent policy platform $y$, a voter decides to support the interest group on the issue $i$ with the largest positive value of $s_{i}\left(x_{i}-y_{i}\right)^{2}$, where $i \in\{1,2\} .{ }^{11}$ In this way the population of voters is divided over the different interest groups. Now consider the interest group located at position $x_{1}$ on the first issue. Identification with the interest group's stance (see the Introduction) stimulates its members, who take the same position on the first issue, to vote for that political candidate which is closest to the interest group on this issue. Thus, if $y$ and $z$ are the policy platforms of the two political parties, then identification is formalized as inducing a member of this interest group to vote for the first party according to the following decision rule ${ }^{12}$

$$
\text { vote for } y \text { if }\left\{\begin{array}{l}
\left|y_{1}-x_{1}\right|<\left|z_{1}-x_{1}\right| \\
\left|y_{1}-x_{1}\right|=\left|z_{1}-x_{1}\right| \text { and }\left|y_{2}-x_{2}\right|<\left|z_{2}-x_{2}\right|
\end{array}\right.
$$

and similarly for the second party. Naturally, we will assume that the voter votes with probability $\frac{1}{2}$ for either policy platform if $|y-x|=|z-x|$. A similar decision rule holds for members of interest groups on the other issue. The introduction of interest groups thus effectively is equivalent with a change in the structure of voter preferences. Similar to $W(y)$ we denote by $W^{I}(y)$ the set of policy platforms defeating the incumbent's platform $y$ when interest groups are present.

The aim of this paper is to investigate the consequences of the introduction of interest groups for the winning sets given an incumbent's position $y$, i.e. the areas of the winning sets denoted by $|W(y)|$ and $\left|W^{I}(y)\right|$. Our main result is

Theorem 1 Let $\underline{s}>0$ be given. Denote

$$
\Psi(\underline{s})=\min \left\{\frac{2(\varphi(\underline{s}))^{\frac{3}{2}}}{1+(\varphi(\underline{s}))^{3}}, \frac{1}{\sqrt{1+4 \sqrt{\underline{s}} \varphi(\underline{s})}}\right\},
$$

where $\varphi(\underline{s})=1+\frac{1}{s}$. Then for all $y \in B(O, \Psi(\underline{s}) K) \backslash\{O\}$ the area of the winning set increases in the presence of interest groups.

This result shows that for all incumbent positions $y$ within a prespecified circle within the issue space, the winning set increases in the presence of the interest

\footnotetext{
${ }^{10}$ For models of interest group formation using a similar approach but applied to, respectively, taxation and informative lobbying, see Sadiraj, Tuinstra, and van Winden $(2005,2006)$.

${ }^{11}$ We assume that a voter with $s_{1}\left(x_{1}-y_{1}\right)^{2}=s_{2}\left(x_{2}-y_{2}\right)^{2}$ with probability $\frac{1}{2}$ chooses to join the interest group on issue 1 and otherwise chooses the interest group on issue 2 .

${ }^{12}$ Effectively, this means that identification leads voters to change their issue weight vector from $s \in$ $\mathcal{S}^{2}$ to either $(1,0)$ or $(0,1)$. Put differently, decisions of interest group members can be represented by lexicographic preferences, instead of by weighted Euclidean distance. This is the sense in which voters identifying with an interest group conform to the issue important to that group.
} 

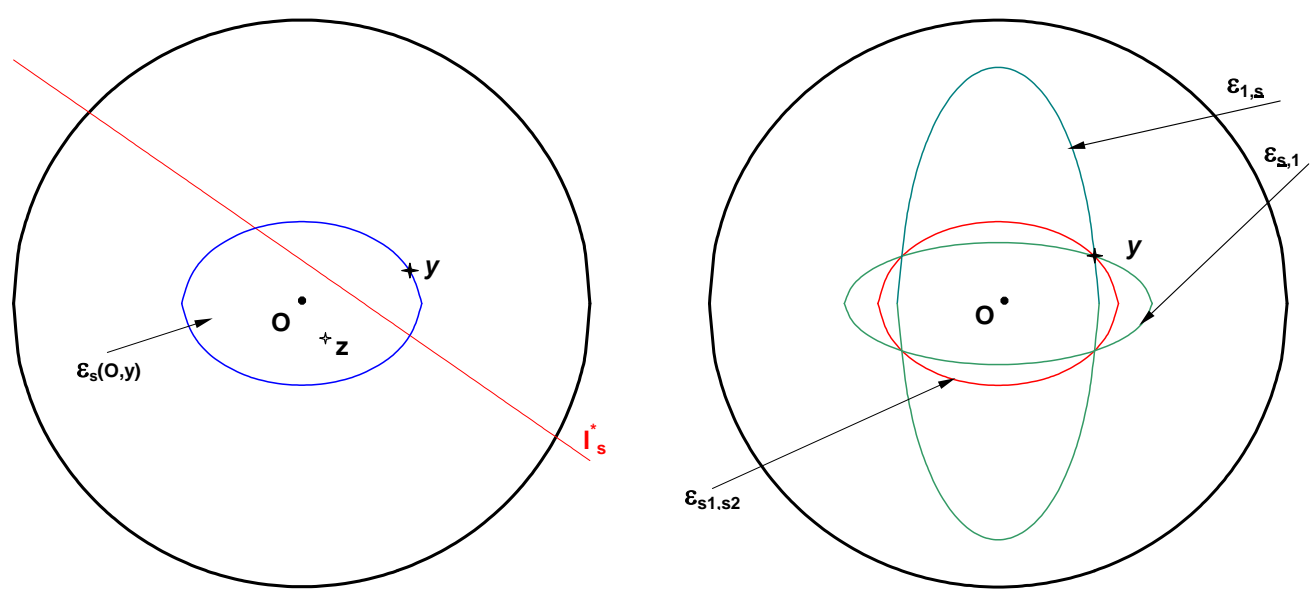

Figure 1: Construction of the winning set for the benchmark model. Left panel shows how $W(y ; s)$ can be constructed (part 2 of Proposition 2), the right panel illustrates part 3 of Proposition 2.

groups. Note that this circle is shrinking as $\underline{s}$ approaches 0 and that the radius of this circle goes to $\frac{1}{3} K$ as $\underline{s}$ approaches 1 . For an intermediate value of $\underline{s}=\frac{1}{2}$, we have $\Psi\left(\frac{1}{2}\right) K=(1+6 \sqrt{2})^{-\frac{1}{2}} K \approx 0.325 K$, which is rather close to $\frac{1}{3} K$ already. Notice that if the incumbent's position falls outside the region specified by Theorem 1, i.e. the incumbent platform is at least a distance $\Psi(\underline{s}) K$ away from the origin, which is the equilibrium point, the winning set for the challenger is relatively large anyway, whether interest groups are present or not.

\section{Outline of the proof of the Theorem}

In this section we will outline the proof of Theorem 1 in a number of intuitive steps. Formal and rigorous proofs of these different steps are relegated to the appendix. It will be convenient to define, for given $v, w \in \mathbb{R}^{2}$ and $c \in \mathcal{S}^{2}$

$$
\mathcal{E}_{c}(v, w)=\left\{x \in \mathbb{R}^{2}:\|x-v\|_{c}^{2}<\|w-v\|_{c}^{2}\right\} .
$$

Hence $\mathcal{E}_{c}(v, w)$ contains all the points inside an ellipse, which is centered at the point $v$, and going through the point $w$. The area of this ellipse equals $\pi\|w-v\|_{c}^{2} / \sqrt{c_{1} c_{2}}$.

First we consider the benchmark model, i.e. electoral competition between political parties in the absence of interest groups. We have the following result.

Proposition 2 Assume voters' ideal positions are independently (across issues and across voters) drawn from the uniform distribution on $\mathcal{X}$ and that voters' weights on each issue are independently drawn from a distribution on $\mathcal{S}$ and are uncorrelated with each other and with the ideal positions. Let $y$ be the platform of the incumbent and let $C=\{(\underline{s}, 1),(1, \underline{s})\}$. Then 
1. $W(O)=\emptyset$,

2. $W(y ; s)=\mathcal{E}_{s}(O, y)$,

3. $\forall y \in \mathcal{X} \backslash\{O\}, W(y) \subset \bigcup_{c \in C} \mathcal{E}_{c}(O, y)$ and $|W(y)| \leq \pi\|y\|^{2} \varphi(\underline{s}) \sqrt{\underline{s}}$.

This result is illustrated in Figure 1. Observe that any line through the origin $O$ divides the issue space $\mathcal{X}$ into two regions which are equally large. Therefore, since ideal points are symmetrically (and uniformly) distributed over the issue space, no other platform will be able to defeat the origin (the origin here corresponds to the position of the generalized median voter, see Hoyer and Mayer, 1974). Now consider an arbitrary position $z$ in $\mathcal{E}_{s}(O, y)$ (see the left panel of Figure 1). The line $l_{s}^{*}$ presents all positions $x$ such that all voters with weight vector $s$ and ideal point $x$ are indifferent between $z$ and $y$. Therefore all voters with weight vector $s$ and an ideal point below $l_{s}^{*}$ will vote for $z$ and it is then easily seen that the majority of voters with that profile will indeed vote for $z$ (basically, since voters with ideal point $O$ will vote for $z$ ). This argument holds if and only if $z$ is an element of $\mathcal{E}_{s}(O, y)$ and therefore $W(y ; s)=\mathcal{E}_{s}(O, y)$. Moreover, for all $s \in \mathcal{S}^{2}$ we have $\mathcal{E}_{s}(O, y) \subset \bigcup_{c \in C} \mathcal{E}_{c}(O, y)$ (see the right panel of Figure 1), which implies $W(y) \subset \bigcup_{c \in C} \mathcal{E}_{c}(O, y)$. With some tedious but straightforward computations one can then derive the upper bound from part 3 of Proposition 2.

Let us now turn to the model with interest groups. We will derive a lower bound for the size of the winning set in the model with interest groups in a number of steps. The results are driven by the fact that decisions of interest group members can now be represented by lexicographic preferences, instead of by weighted Euclidean distance. Consider all voters with a certain weight vector $s \in \mathcal{S}^{2}$. Consider a given position $y$ of the incumbent. From now on we will, without loss of generality, assume $y_{1} \geq 0$ and $y_{2} \geq 0$. In order to determine the sizes of the interest groups take the two lines $l_{-}$and $l_{+}$, which go through $y$ with slopes $-\sqrt{\frac{s_{1}}{s_{2}}}$ and $\sqrt{\frac{s_{1}}{s_{2}}}$, respectively. These two lines demarcate four regions in the issue space (see Figure 2), which we denote as $I$ (below $y$ ), II (above $y$ ), III (to the right of $y$ ) and $I V$ (to the left of $y$ ). For each of the regions it is easy to determine whether voters with an ideal point in that region (and with weight vector $s$ ) will join an interest group on the first issue or one on the second issue. The following lemma (the proof of which is left to the reader) summarizes this.

Lemma 3 Consider voter $j$ with ideal point $x$ and weight vector $s$.

i) If $x \in I \cup I I$ then voter $j$ joins the interest group on the second issue.

ii) If $x \in I I I \cup I V$ then voter $j$ joins the interest group on the first issue.

Figure 2 also illustrates the lines $l l_{-}$and $l l_{+}$, which pass through the origin $O$ and lie parallel to $l_{-}$and $l_{+}$, and the regions $S_{+}(y ; s)$ and $S_{-}(y ; s)$ which lie between $l_{+}$ 


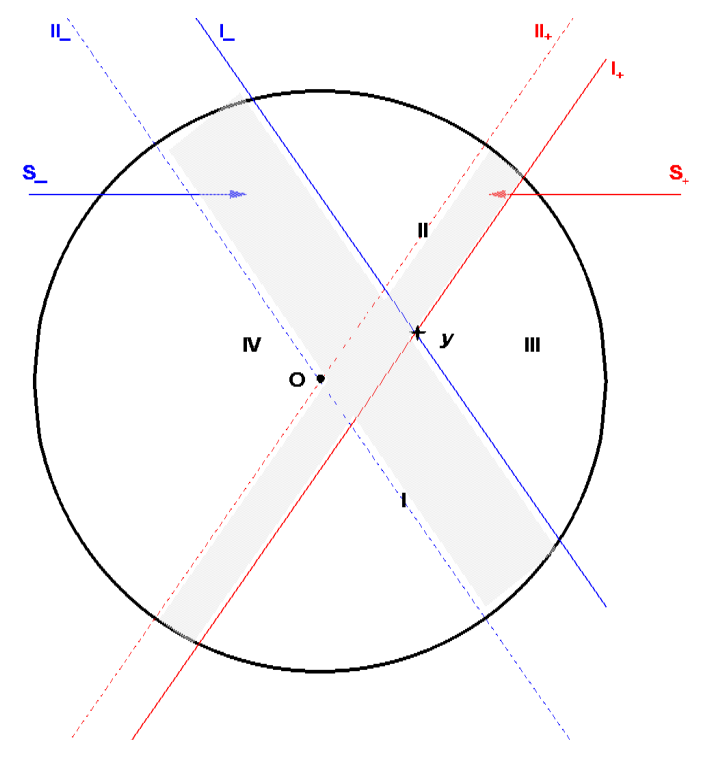

Figure 2: Illustration of the different regions needed for determining the area of the winning set for the interest group model. The point $y$ corresponds to the incumbent's position. The lines $l_{-}$and $l_{+}$through $y$ divide the issue space in four regions: regions $I$ and $I I$ (below and above $y$ respectively, containing ideal positions of voters joining interest groups on the second issue, see Lemma 3) and the regions $I I I$ and $I V$ (to the right and to the left of $y$, respectively, containing the ideal positions of voters joining interest groups on the first issue). The shaded region between $l_{-}$and $l l_{-}$(between $l_{+}$ and $\left.l l_{+}\right)$corresponds to $S_{-}(y ; s)\left(S_{+}(y ; s)\right)$.

and $l l_{+}$and between $l_{-}$and $l l_{-}$, respectively. The significance of these two regions will become evident shortly.

We are now ready to prove our main results. First, in Proposition 4 we characterize, given the incumbent's position $y \neq 0$ and a weight vector $s$, the set of positions for the challenger that attract more than half of the voters with that weight vector. Proposition 5 then looks at the intersection of all these sets over different weight vectors, in order to find a lower bound for $\left|W^{I}(y)\right|$. Finally, comparing this lower bound with the upper bound for $|W(y)|$ that was found in Proposition 2, Theorem 1 is proven.

Proposition 4 In the presence of interest groups any element of the set $A(y ; s)$ is supported by more than half of the voters with weight vector $s$, where $A(y ; s)=$ $A_{-}(y ; s) \cup A_{+}(y ; s)$ and

$$
\begin{aligned}
& A_{-}(y ; s)=\left\{z: z_{1}<y_{1}, z_{2}<y_{2}\right\} \cap \mathcal{E}_{s}\left(y, w_{-}\right) \cap \mathcal{X}, \\
& A_{+}(y ; s)=\left\{z: z_{1}<y_{1}, z_{2} \geq y_{2}\right\} \cap \mathcal{E}_{s}\left(y, w_{+}\right) \cap \mathcal{X} .
\end{aligned}
$$

where $w_{*}, * \in\{-,+\}$ satisfies $\left\|y-w_{*}\right\|_{s}^{2}=4 \sqrt{s_{1} s_{2}}\left|S_{*}(y ; s)\right|$. 

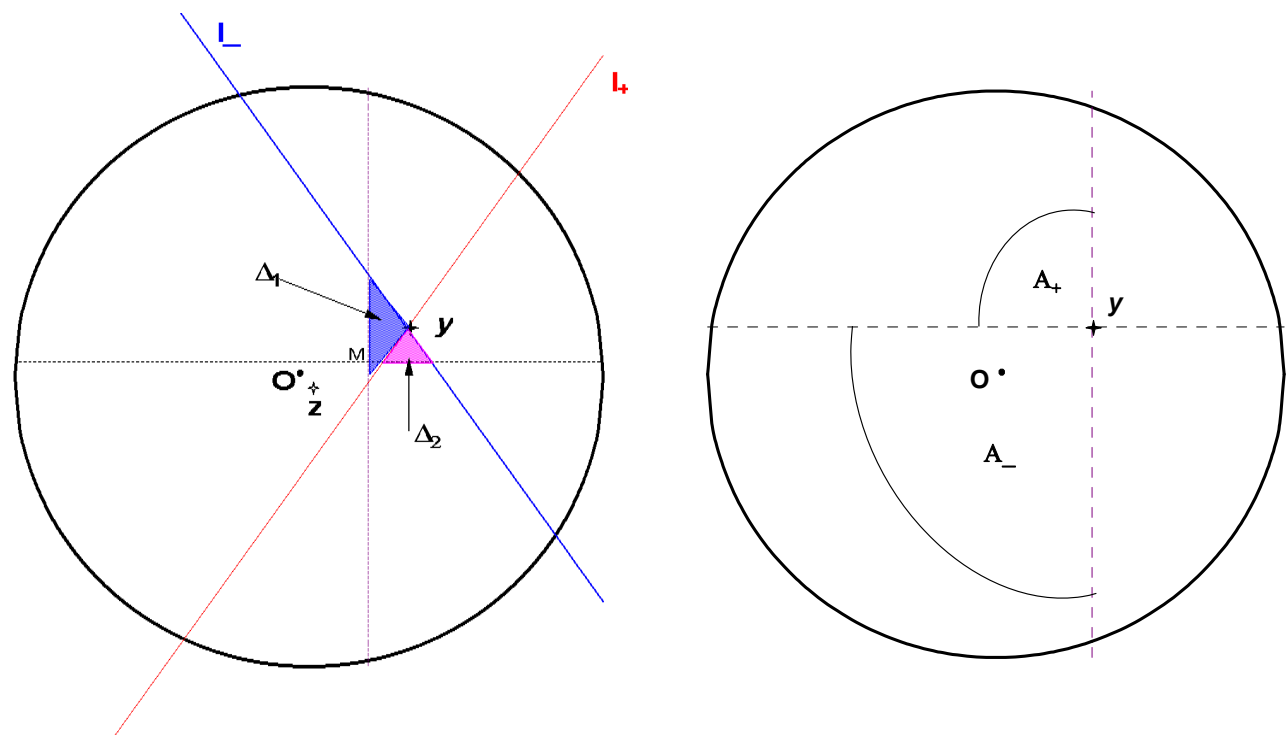

Figure 3: Illustration of Proposition 4. The left panel shows how the set $A_{-}(y ; s)$ can be constructed, the right panel show what the sets $A_{-}(y ; s)$ and $A_{+}(y ; s)$ look like.

This proposition tells us that the area of $S_{-}(y ; s)$ (or $S_{+}(y ; s)$ ) defines a region $A_{-}(y ; s)$ (or $\left.A_{+}(y ; s)\right)$, which is part of an ellipse and has the property that all positions within this region defeat the incumbent for the given weight profile. The proof of this proposition can be illustrated by the left panel in Figure 3. Pick an arbitrary $z<y$ and denote by $M$ the midpoint of the line connecting $z$ with the incumbent's position $y$. Now draw a vertical and a horizontal line through $M$. The intersections of these lines with $l_{+}$and $l_{-}$define two triangles, $\triangle_{1}$ and $\triangle_{2}$. By construction (see Lemma 3 ) all voters with weight profile $s$ and an ideal point below $l_{-}$will vote for $z$, except those voters with ideal points in one of the two triangles. Hence we have to determine for which positions $z$ the area of the associated triangles is not too large. In fact, from Figure 2 it follows that the area below $l_{-}$can be described as $\left|l_{-}(O)\right|=\frac{1}{2}|\mathcal{X}|+\left|S_{-}(y ; s)\right|$. Moreover, the area corresponding to the voters that cast their vote for the incumbent is given by $|\mathcal{X}|-\left(\left|l_{-}(O)\right|-\left|\triangle_{1}+\triangle_{2}\right|\right)$. Using the expression for $\left|l_{-}(O)\right|$ we find that the challenger wins the election when $\left|\triangle_{1}+\triangle_{2}\right|<\left|S_{-}(y ; s)\right|$. This inequality holds exactly for all $z \in A_{-}(y ; s)$. A similar argument can be made for $A_{+}(y ; s)$. The sets $A_{-}(y ; s)$ and $A_{+}(y ; s)$ are illustrated in the right panel of Figure 3.

The set $A(y ; s)$ can be constructed for any weight profile $s$. Clearly, a policy position $z$ which lies in the intersection $\cap_{s} A(y ; s)$ of these sets has the property that for each possible weight profile a majority of the voters with this profile will vote for that policy position $z$. Any element of this set will therefore belong to the winning set $W^{I}(y)$ and the area of this intersection then gives a lower bound for $\left|W^{I}(y)\right|$. The next proposition deals with this intersection and a lower bound for its area, for the case with $y_{1}>0$ and $y_{2}>0$. For the other configurations of $y$ a similar result can be obtained.

Proposition 5 Consider $y_{1}>0$ and $y_{2}>0$. Let $R_{y}=2 \sqrt{\underline{S}(y) \sqrt{\underline{s}}}$, where $\underline{S}(y)=$ 
$\inf \left\{\left|S_{-}(y ; s)\right|: s_{1}, s_{2} \in \mathcal{S}\right\}$

1. $B=B\left(y, R_{y}\right) \cap\left\{z \in \mathcal{X}: z_{i}<y_{i}, i=1,2\right\} \subset \cap_{s} A(y ; s)$;

2. $\underline{S}(y) \geq\left(K+\sqrt{K^{2}-\|y\|^{2}}\right) \frac{\left(\left|y_{1}\right|+\left|y_{2}\right|\right)}{\sqrt{\varphi(\underline{s})}}$.

We now have determined an upper bound for the area of the winning set in the absence of interest groups (Proposition 2) and a lower bound for the area of the winning set in the presence of interest groups (Proposition 5). Our main theorem then simply follows from comparing these two bounds.

Sketch of the proof of Theorem 1. From Proposition 2, part 3 we find that

$$
|W(y)| \leq \pi\|y\|^{2} \varphi(\underline{s}) \sqrt{s} .
$$

Moreover, Proposition 5 implicitly gives a lower bound for $\left|W^{I}(y)\right|$. Comparing the two, we find that a sufficient condition for $\left|W^{I}(y)\right|>|W(y)|$ is that $y$ belongs to $B(O, \Psi(\underline{s}) K) \backslash\{O\}$, where

$$
\Psi(\underline{s})=\min \left\{\frac{2(\varphi(\underline{s}))^{\frac{3}{2}}}{1+(\varphi(\underline{s}))^{3}}, \frac{1}{\sqrt{1+4 \varphi(\underline{s}) \sqrt{\underline{s}}}}\right\} .
$$

\section{Concluding remarks}

In this short paper we have shown that the introduction of interest groups into a spatial voting model of electoral competition between two political parties will typically increase the winning set for the challenger. Since in mass elections candidates or political parties will in general lack information concerning the distribution of voter preferences, it can be quite difficult to find winning positions. This paper suggests that winning sets for the challenger will increase in the presence of interest groups, thereby increasing the probability of winning for the challenger and making it harder to locate an equilibrium point if it exists. This effect, which is driven by the (empirically plausible) assumption that interest group membership is positively related to the discontent with the incumbent's policy on the relevant issue has, to our knowledge, never been shown before. The effect is confirmed in a simulation study by Sadiraj, Tuinstra and van Winden (2006). Moreover, this simulation study indicates that, because of the increase in the winning set in the presence of interest groups, separation between policy platforms increases and the rate of convergence of policy platforms to the equilibrium point decreases.

The model we have chosen to present our main result is, of course, highly stylized. Two final remarks are in order here. First, as can be seen from Section 3, we have been quite conservative in providing upper and lower bounds of the relevant areas. We therefore expect that our main result holds for a significantly larger set than the 
one specified by Theorem 1. Secondly, for matters of exposition we chose as issue space a ball around the origin, where a rectangular issue space is more common in the literature. Actually, the issue space might be larger than this ball. A distribution of voter preferences over for example a square or rectangle would substantively give the same results, but would lead to a cumbersome written proof. The present assumption is also motivated by the observation that voters typically do not take extreme positions on all issues. What the precise effect of interest groups will be under a more general specification of the (dimension of the) issue space, the distribution of ideal points and the structure of voter preferences or in the absence of an equilibrium point is left for future research. Note however that the result presented here holds for any distribution of strengths, provided that this distribution is independent of the ideal points. The main message here is that (through identification) interest groups will have an impact on voting behavior and thereby influence the political decision making process, even apart from their influence on the latter through lobbying, campaign contributions or endorsements, which are the channels studied in the existing political economic literature on interest groups.

\section{References}

[1] Akerlof, G.A. and R.E. Kranton (2000): Economics and identity. Quarterly Journal of Economics 115, 715-753.

[2] Austen-Smith, D. (1994): Interest groups: money, information and influence. In: D.C. Mueller (ed.), Perspectives on Public Choice, Cambridge: Cambridge University Press.

[3] Baumeister, R. F., E. Bratslavsky, C. Finkenauer, and K. D. Vohs (2001): Bad is stronger than good. Review of General Psychology 5, 323-370.

[4] Brewer, M.B. (1979): In-group bias in the minimal intergroup situation: a cognitive-motivational analysis, Psychological Bulletin 86, 307-324.

[5] Chong, D. (1991): Collective Action and the Civil Rights Movement. Chicago: University of Chicago Press.

[6] Congleton, R.D., A.L. Hillman and K.A. Konrad (Eds.) (2008): 40 Years of Research on Rent Seeking (2 volumes). Berlin: Springer

[7] Enelow, J.M. and M.J. Hinich (1984): The Spatial Theory of Voting: An Introduction. Cambridge: Cambridge University Press.

[8] Festinger, L. and E. Aronson (1968): Arousal and reduction of dissonance in social contexts. In: D. Cartwright and A. Zander (eds.), Group Dynamics, New York: Harper \& Row.

[9] Grossman, G.M. and E. Helpman (2001): Special Interest Politics. Cambridge: The MIT Press. 
[10] Hoyer, R.W. and L.S. Mayer (1974): Comparing strategies in a spatial model of electoral competition. American Journal of Political Science 18, 501-523.

[11] Javeline, D. (2003): The role of blame in collective action: evidence from Russia. American Political Science Review 97, 107-121.

[12] Kernell, S. (1977): Presidential popularity and negative voting. American Political Science Review 71, 44-66.

[13] Kollman, K., J.H. Miller and S.E. Page (1992): Adaptive parties in spatial elections. American Political Science Review 86, 929-937.

[14] Kramer, G.H. (1977): A dynamic model of political equilibrium. Journal of Economic Theory 16, 310-334.

[15] Lau, R.R. (1982): Negativity in political perception. Political Behavior 4, 353378.

[16] Offerman, T. (2002): Hurting hurts more than helping helps. European Economic Review 46, 1423-1437.

[17] Plott, C.R. (1967): A notion of equilibrium and its possibility under majority rule. American Economic Review 57, 787-806.

[18] Potters, J., and R. Sloof (1996): Interest groups: a survey of empirical models that try to assess their influence. European Journal of Political Economy 12, 403-442.

[19] Prat, A. (2006): Rational voters and political advertising. In: B.R. Weingast and D.A. Wittman (Eds.), The Oxford Handbook of Political Economy. Oxford: Oxford University Press.

[20] Richardson, J.J. (ed.) (1994): Pressure Groups. New York: Oxford University Press.

[21] Romer, P. (1996): Preferences, promises, and the politics of entitlement. In: V. Fuchs (ed.), Individual Social Responsibility, Chicago: University of Chicago Press.

[22] Sadiraj, V., J. Tuinstra and F. van Winden (2005): Interest group size dynamics and policymaking. Public Choice 125, 271-303.

[23] Sadiraj, V., J. Tuinstra and F. van Winden (2006): A computational electoral competition model with social clustering and endogenous interest groups as information brokers. Public Choice 129, 169-187.

[24] Schram, A. and J. Sonnemans (1996): Voter turnout as a participation game: An experimental investigation. International Journal of Game Theory 25, 385-406. 
[25] Shayo, M. (2009): A model of social identity with an application to political economy: Nation, class, and redistribution. American Political Science Review $103,147-174$.

[26] Tajfel, H. and J.C. Turner (1986): The social identity theory of intergroup behavior. In: S. Worchel and W.G. Austin (eds.), Psychology of Intergroup Relations, Chicago: Nelson-Hall.

[27] Tovey, C.A. (2010): A critique of distributional analysis in the spatial model. Mathematical Social Sciences 59, 88-101.

[28] van Winden, F. (2003): Interest group behavior and influence. In: C.K. Rowley and F. Schneider (eds.), Encyclopedia of Public Choice, Boston: Kluwer Academic Publishers.

\section{A Proofs}

This appendix contains formal proofs for the results in Section 3.

\section{Proof of Proposition 2.}

1. By symmetry, any line through the origin $O$ divides the issue space $\mathcal{X}$ into two regions which are equally large. The uniform distribution then implies the same expected number of voters on either side of such a line. Therefore the origin corresponds to the position of the generalized median voter and no position will strictly defeat it. Hence $W(O)=\emptyset$.

2. Without loss of generality we assume $y_{1} \geq y_{2}>0$. Consider an arbitrary policy position $z$, with $z_{i} \leq y_{i}, i=1,2$. We want to determine under which conditions $z \in W(y)$. Let us first determine all positions $x^{*}$ such that voters with the considered weight profile $s$ are indifferent between $z$ and $y$. These positions $x^{*}$ have to satisfy

$$
\left\|y-x^{*}\right\|_{s}^{2}=\left\|z-x^{*}\right\|_{s}^{2} .
$$

Solving for $x_{2}^{*}$ is straightforward and gives

$$
x_{2}^{*}=\frac{-2 s_{1}\left(y_{1}-z_{1}\right) x_{1}^{*}+\|y\|_{s}^{2}-\|z\|_{s}^{2}}{2 s_{2}\left(y_{2}-z_{2}\right)} .
$$

Let us denote the line defined by (3) as $l_{s}^{*}$. Clearly, $l_{s}^{*}$ separates the issue space into two regions and all voters in the region below and to the left of $l_{s}^{*}$ vote for the challenger, if it selects position $z$. Now suppose $l_{s}^{*}$ cuts the vertical axis at some positive value. Then we can draw a line that goes through $O$ and that lies parallel to $l_{s}^{*}$. This line through $O$ divides $\mathcal{X}$ into two equal regions, implying that the region lying below and to the left of $l_{s}^{*}$ will be larger than the region above and to the right of $l_{s}^{*}$. Therefore, the challenger will win at $z$ if $l_{s}^{*}$ intersects the vertical axis at some positive value. This condition reduces to

$$
\|y\|_{s}^{2}-\|z\|_{s}^{2}>0
$$


which defines the ellipse given in the proposition. In a similar fashion the same condition can be derived for positions $z$ with $z_{1} \leq y_{1}$ and $z_{2}>y_{2}$ and for positions $z$ with $z_{1}>y_{1}$ and $z_{2} \leq y_{2}$. It should be clear that positions $z$ with $z_{1} \geq y_{1}$ and $z_{2} \geq y_{2}$ never defeat the incumbent.

3. First, we show that $W(y) \subset \bigcup_{c \in C} \mathcal{E}_{c}(O, y)$. A sufficient condition for this to be true is $\cup_{s} \mathcal{E}_{s}(O, y) \subset \cup_{c} \mathcal{E}_{c}(O, y)$.

Let $x \in \cup_{s} \mathcal{E}_{s}(O, y)$. Hence, $x \in \mathcal{E}_{s}(O, y)$ for some $s_{1}, s_{2} \in \mathcal{S}$ and therefore $\|x\|_{s}^{2}<\|y\|_{s}^{2}$, or equivalently

$$
\left(x_{1}^{2}-y_{1}^{2}\right)<\frac{s_{2}}{s_{1}}\left(y_{2}^{2}-x_{2}^{2}\right) \text {. }
$$

Note that $\underline{s} \leq \frac{s_{2}}{s_{1}} \leq \frac{1}{s}$, for all $s_{1}, s_{2} \in \mathcal{S}$. If $\left|x_{2}\right| \leq\left|y_{2}\right|$, then

$$
\left(x_{1}^{2}-y_{1}^{2}\right)<\frac{s_{2}}{s_{1}}\left(y_{2}^{2}-x_{2}^{2}\right) \leq \frac{1}{\underline{s}}\left(y_{2}^{2}-x_{2}^{2}\right) \Longleftrightarrow \underline{s} x_{1}^{2}+x_{2}^{2} \leq \underline{s} y_{1}^{2}+y_{2}^{2}
$$

and therefore $x \in \mathcal{E}_{c}(O, y)$ with $c=(\underline{s}, 1)$. On the other hand, if $\left|x_{2}\right|>\left|y_{2}\right|$ we have

$$
\left(x_{1}^{2}-y_{1}^{2}\right)<\frac{s_{2}}{s_{1}}\left(y_{2}^{2}-x_{2}^{2}\right) \leq \underline{s}\left(y_{2}^{2}-x_{2}^{2}\right),
$$

and therefore $x \in \mathcal{E}_{c}(O, y)$ with $c=(1, \underline{s})$.

Second, we calculate the area of $\bigcup_{c \in C} \mathcal{E}_{c}(O, y)$. Recall that the area of $\mathcal{E}_{c}(O, y)$ is given by $\pi \frac{\|y\|_{c}^{2}}{\sqrt{c_{1} c_{2}}}$ and therefore we have

$$
\begin{aligned}
\left|\bigcup_{c \in C} \mathcal{E}_{c}(O, y)\right| & \leq \sum_{c \in C} \pi \frac{\|y\|_{c}^{2}}{\sqrt{c_{1} c_{2}}} \\
& =\frac{\pi}{\sqrt{\underline{s}}}\left(\|y\|_{(\underline{s}, 1)}^{2}+\|y\|_{(1, \underline{s})}^{2}\right) \\
& =\pi \varphi(\underline{s}) \sqrt{\underline{s}}\|y\|^{2}
\end{aligned}
$$

Hence,

$$
|W(y)| \leq\left|\bigcup_{c \in C} \mathcal{E}_{c}(O, y)\right| \leq \pi \varphi(\underline{s}) \sqrt{\underline{s}}\|y\|^{2} .
$$

Proof of Proposition 4. Consider a position $z \in A_{-}(y ; s)$. Denote by $M=$ $\left(M_{1}, M_{2}\right)=\left(\frac{y_{1}+z_{1}}{2}, \frac{y_{2}+z_{2}}{2}\right)$ the midpoint of the line connecting $z$ with the incumbent's position $y$. Now draw a vertical and a horizontal line through $M$ and consider the intersections of these lines with $l_{+}$and $l_{-}$. Denote these intersections by $i_{*}$, where $* \in\{+,-\}$ and $i \in\{1,2\}$. Hence $1_{+}\left(1_{-}\right)$is the intersection between the vertical line 
through $M$ and $l_{+}\left(l_{-}\right)$and $2_{+}\left(2_{-}\right)$is the intersection between the horizontal line through $M$ and $l_{+}\left(l_{-}\right)$. Now consider the subspace $\mathcal{L} \equiv\left(l_{-}(O)-\left(1_{-} 1_{+} 2_{+} 2_{-} 1_{-}\right)\right) \cap \mathcal{X}$. From Lemma 3 we know that all voters in $I$ join the interest group on the second issue and all voters in $I V$ join the interest group on the first issue. For the voters in $I \cap \mathcal{L}$ we have $\left|x_{2}^{j}-z_{2}\right|<\left|x_{2}^{j}-y_{2}\right|$ and for all voters in $I V \cap \mathcal{L}$ we have $\left|x_{1}^{j}-z_{1}\right|<\left|x_{1}^{j}-y_{1}\right|$. This proves that the challenger at position $z$ gets votes from voters with ideal positions in $\mathcal{L}$. Hence it gets more than half of the votes if

$$
\left|S_{-}\right|>\left|1_{-} 1_{+} 2_{+} 2_{-} 1_{-}\right|
$$

since $\left|S_{-}\right|=\left|l_{-}(O)\right|-\frac{1}{2}|\mathcal{X}|$.

First, denote $\triangle_{1}$ the triangle $y 1_{+} 1_{-}$and $\triangle_{2}$ the triangle $y 2_{+} 2_{-}$and note that

$$
\begin{aligned}
\left|\left(1_{-} 1_{+} 2_{+} 2_{-} 1_{-}\right) \cap \mathcal{X}\right| & \leq\left|\triangle_{1}\right|+\left|\triangle_{2}\right| \\
& =\sqrt{\frac{s_{1}}{s_{2}}}\left(y_{1}-M_{1}\right)^{2}+\sqrt{\frac{s_{2}}{s_{1}}}\left(y_{2}-M_{2}\right)^{2} \\
& =\frac{1}{4} \sqrt{\frac{s_{1}}{s_{2}}}\left(y_{1}-z_{1}\right)^{2}+\frac{1}{4} \sqrt{\frac{s_{2}}{s_{1}}}\left(y_{2}-z_{2}\right)^{2} \\
& \leq\left|S_{-}\right|
\end{aligned}
$$

The first inequality follows from the fact that some parts of the triangles $\triangle_{i}, i=1,2$ might not be in $\mathcal{X}$, the first equal sign follows from computation of the surface of the two triangles, the second equality follows from the definition of the midpoint $M$ and the final step follows from the fact that $z \in A_{-}(y ; s)$. A similar reasoning holds for $A_{+}(y ; s)$. Thus, it is shown that all positions $z \in A(y ; s)$ are expected to defeat the incumbent. 


\section{Proof of Proposition 5.}

1. First, we show that

$$
B \subset \cap_{s} A(y ; s)
$$

Indeed, let $x \in B$ i.e. $x_{i}<y_{i}$ and

$$
\|x-y\|^{2} \leq R_{y}^{2}=4 \underline{S} \sqrt{\underline{s}}
$$

Thus, $\forall s \in \mathcal{S}^{2}$ and $c=\left(\frac{1}{4} \sqrt{\frac{s_{1}}{s_{2}}}, \frac{1}{4} \sqrt{\frac{s_{2}}{s_{1}}}\right)$

$$
\begin{aligned}
\|x-y\|_{c}^{2} & \leq \frac{1}{4} \sqrt{\frac{1}{\underline{s}}}\|x-y\|^{2} \\
& \leq \frac{1}{4} \sqrt{\frac{1}{\underline{s}}} 4 \underline{S} \sqrt{\underline{s}} \\
& \leq\left|S_{-}(y ; s)\right|
\end{aligned}
$$

and therefore, $x \in A_{-}(y ; s) \subset A(y ; s)$.

2. The next step consists of deriving a lower bound for the radius of $B$. First, let $d\left(O, l_{+}\right)\left(d\left(O, l_{-}\right)\right)$correspond to the distance of the origin from the line $l_{+}$ $\left(l_{-}\right)$, i.e.

$$
d\left(O, l_{+}\right)=\min \left\{\|x\|: x \in l_{+}\right\} .
$$

For the case we are considering $\left(y_{1}>0\right.$ and $\left.y_{2}>0\right)$, let $h(y ; s)=d\left(O, l_{-}\right)$ (which means that we are focussing on $A_{-}(y ; s)$ ). For all $s \in \mathcal{S}^{2}$, one has

$$
\begin{aligned}
\left|S_{-}(y, s)\right| & =2 \int_{0}^{h(y ; s)} \sqrt{K^{2}-z^{2}} d z \\
& =\left[z \sqrt{K^{2}-z^{2}}+K^{2} \arcsin \frac{z}{K}\right]_{0}^{h(y ; s)} \\
& =h(y ; s) \sqrt{K^{2}-h(y ; s)^{2}}+K^{2} \arcsin \frac{h(y ; s)}{K} .
\end{aligned}
$$

By definition we have $h(y ; s) \leq\|y\| \leq K$. Furthermore, $\arcsin x>x$ for all $x \in(0,1]$. Using these properties we find

$$
\left|S_{-}(y, s)\right| \geq \underline{h}(y)\left(\sqrt{K^{2}-\|y\|^{2}}+K\right),
$$

where $\underline{h}(y)=\inf _{s_{1, s_{2} \in \mathcal{S}}} h(y ; s)$. Finally, since $\underline{s} \leq \min \left\{s_{1} / s_{2}, s_{2} / s_{1}\right\}$ and therefore $\frac{\sqrt{\underline{s}}}{\sqrt{1+\underline{s}}} \leq \min \left\{\frac{\sqrt{s_{1}}}{\sqrt{s_{1}+s_{2}}}, \frac{\sqrt{s_{2}}}{\sqrt{s_{1}+s_{2}}}\right\}$, we have

$$
\begin{aligned}
h(y ; s) & =\frac{\sqrt{s_{1}}\left|y_{1}\right|+\sqrt{s_{2}}\left|y_{2}\right|}{\sqrt{s_{1}+s_{2}}} \\
& \geq \frac{\sqrt{\underline{s}}}{\sqrt{1+\underline{s}}}\left(\left|y_{1}\right|+\left|y_{2}\right|\right)
\end{aligned}
$$


which implies $\underline{h}(y) \geq \frac{\sqrt{\underline{s}}\left(\left|y_{1}\right|+\left|y_{2}\right|\right)}{\sqrt{1+\underline{s}}}$. Using $\varphi(s)=1+1 / s$ one now has

$$
\left|S_{-}(y ; s)\right| \geq \sqrt{1 / \varphi(\underline{s})}\left(\sqrt{K^{2}-\|y\|^{2}}+K\right)\left(\left|y_{1}\right|+\left|y_{2}\right|\right)
$$

Proof of Theorem 1. Let $\underline{s}$ be given. From Proposition 2 we know that

$$
|W(y)| \leq \pi\|y\|^{2} \sqrt{s} \varphi(\underline{s}) .
$$

From Proposition 5 we know that all positions $z$ in $B(y, 2 \sqrt{\sqrt{\underline{s}}}|\underline{S}|)$, with $z_{1}<y_{1}$ and $z_{2}<y_{2}$ are contained in $W^{I}(y)$. Furthermore we found that

$$
\left|S_{-}(y, s)\right| \geq \underline{S}(y) \geq \sqrt{1 / \varphi(\underline{s})}\left(K+\sqrt{K^{2}-\|y\|^{2}}\right)\left(\left|y_{1}\right|+\left|y_{2}\right|\right) .
$$

If this area lies in $\mathcal{X}$ we know that it presents a lower bound for $\left|W^{I}(y)\right|$. This does not necessarily have to be the case. Let us first compute the two points that have one of the coordinates equivalent with the point $y$ but that lie on the border of $\mathcal{X}$. These points are $\left(y_{1}, \sqrt{K^{2}-y_{1}^{2}}\right)$ and $\left(\sqrt{K^{2}-y_{2}^{2}}, y_{2}\right)$. Now consider the following ball $B(y, r)$ where $r=\min \left\{\left|y_{1}\right|+\sqrt{K^{2}-y_{2}^{2}},\left|y_{2}\right|+\sqrt{K^{2}-y_{1}^{2}}\right\}$. One fourth of this ball lies in $\mathcal{X}$ completely. Therefore there are two possibilities. This fourth part of the ball contains $A_{-}$or is contained in $A_{-}$. So we have to take the minimum of the two lower bounds as a lower bound for $\left|W^{I}(y)\right|$. Consider the first case. Then

$$
\left|W^{I}(y)\right| \geq \pi \sqrt{\underline{s} / \varphi(\underline{s})}\left(K+\sqrt{K^{2}-\|y\|^{2}}\right)\left(\left|y_{1}\right|+\left|y_{2}\right|\right)
$$

and

$$
|W(y)|<\pi\|y\|^{2} \sqrt{s} \varphi(\underline{s})
$$

So we find that, in the presence of interest groups, the size of the winning set is expected to increase for all $y$ satisfying $\left|W^{I}(y)\right| \geq|W(y)|$ or

$$
\pi \sqrt{\underline{s} / \varphi(\underline{s})}\left(K+\sqrt{K^{2}-\|y\|^{2}}\right)\left(\left|y_{1}\right|+\left|y_{2}\right|\right) \geqslant \pi\|y\|^{2} \sqrt{\underline{s}} \varphi(\underline{s})
$$

Using $\left|y_{1}\right|+\left|y_{2}\right| \geq\|y\|$, and rewriting we find that for all $y$ satisfying

$$
\|y\| \leq \frac{2(\varphi(\underline{s}))^{\frac{3}{2}}}{1+(\varphi(\underline{s}))^{3}} K
$$

our property holds. Defining $D_{1} \equiv \frac{2(\varphi(\underline{s}))^{\frac{3}{2}}}{1+(\varphi(\underline{s}))^{3}} K$, the property holds for all $y \in B\left(0, D_{1}\right)$. 
Now consider the second case with

$$
\left|W^{I}(y)\right| \geq \frac{\pi}{4}\left(\min \left\{\left|y_{1}\right|+\sqrt{K^{2}-y_{2}^{2}},\left|y_{2}\right|+\sqrt{K^{2}-y_{1}^{2}}\right\}\right)^{2} .
$$

Suppose, without loss of generality, that $\left|y_{1}\right|+\sqrt{K^{2}-y_{2}^{2}} \leq\left|y_{2}\right|+\sqrt{K^{2}-y_{1}^{2}}$. We look for $\|y\|$ such that

$$
\frac{\pi}{4}\left(\left|y_{1}\right|+\sqrt{K^{2}-y_{2}^{2}}\right)^{2} \geq \pi\|y\|^{2} \sqrt{\underline{s}} \varphi(\underline{s}) .
$$

Again, we will try to derive a condition on $\|y\|^{2}$. Using $0 \leq\left|y_{2}\right|^{2} \leq\|y\|^{2}$, we get

$$
\left|y_{1}\right|^{2}+K^{2}-y_{2}^{2}+2\left|y_{1}\right| \sqrt{K^{2}-y_{2}^{2}} \geq K^{2}-\|y\|^{2} \geq 4\|y\|^{2} \varphi(\underline{s}) \sqrt{\underline{s}}
$$

or

$$
\|y\|^{2} \leq \frac{K^{2}}{1+4 \varphi(\underline{s}) \sqrt{\underline{s}}}
$$

That is, for all $y \in B\left(0, D_{2}\right)$, with $D_{2} \equiv \frac{K}{\sqrt{1+4 \varphi(\underline{s}) \sqrt{\underline{s}}}}$. This concludes the proof of Theorem 1. 